Michele Costa, Attilio Gardini, Paolo Paruolo

\title{
A reduced rank regression approach to tests of asset pricing
}

\section{Serie Ricerche n. 5}

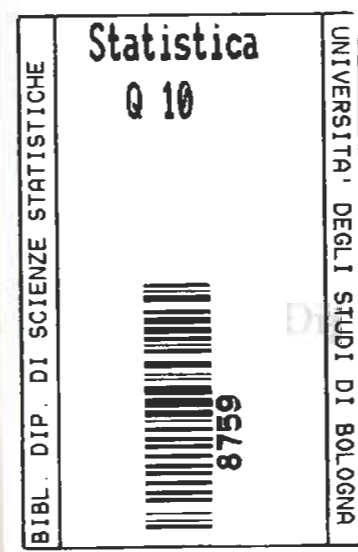
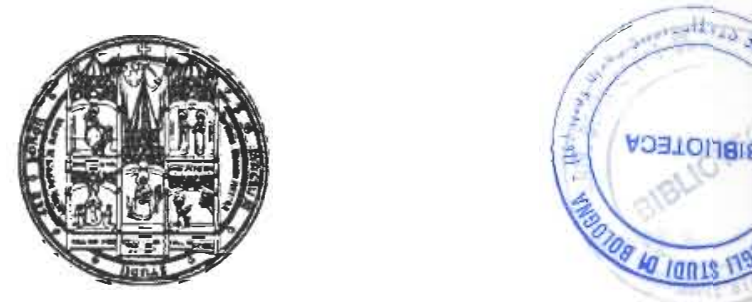

artimento di Scienze Statistiche "Paolo Fortunati"

Università degli studi di Bologna 1992 


\section{1 - Introduction}

Several financial models specify a factor structure for asset returns. The question of unobservability of such factors ${ }^{1}$ has favoured the application of exploratory factor analysis, leaving the interpretation of factors to the ex post empirical analysis of their correlation with observable macroeconomic indicators ${ }^{2}$. In this paper we derive some implications of factor models on observable variables, both from financial and non-financial markets. From a factor model it is in fact possible to derive reduced form models, involving either only asset returns or macroeconomic variables and asset returns, i.e. observable variables. Within such models it is possible to make inference on the number of latent factors. Testing for the number of latent factors makes it possible to test competing models such as the Capital Asset Pricing Model (CAPM) and the Arbitrage Pricing Theory (APT).

Moreover the models involving asset returns and macroeconomic variables can be exploited to estimate the relation of a specific economic indicator with financial latent factors and to test its relevance, thus assessing the connection between financial markets and the rest of the economy.

All the models are special cases of the reduced rank regression model of Anderson (1951), i.e. regression models where the regression coefficient matrix is not full rank (reduced rank) and its rank is equal to the number of latent factors. The gaussian maximum likelihood problem for these models has an analytic solution linked to canonical correlation analysis, and thus favours maximum likelihood estimation and likelihood ratio testing. Although gaussian maximum likelihood can be reinterpreted as a special generalized method of moments or pseudo maximum likelihood solution, the choice of likelihood ratio tests is not robust to the misspecification of the probability model. Extension to robust procedures are not analyzed in this paper, and appear an interesting area for future research. Besides the present approach provides a simple testing strategy which is optimal in the analyzed model.

* This paper in the outcome of a joint project of the authors, who thank CNR (grant n. 2090288) and MURST 40\% 1989-90 «Variabili macroeconomiche, rischio e prezzi delle attività finanziarie» for financial support; for official purposes only we specify that sections 5,6 were written by M. Costa, sections 1,2 by A. Gardini and sections 3 and 4 by P. Paruolo.

1 Roll's critique, for instance, has focused on the unobservability of the market portfolio of Capital Asset Pricing Models, see e.g. Gibbons and Ferson (1985). 2 A notable exception is the line of models initiated by Gibbons and Ferson (1985), see also Ferson (1990). 
The rest of the paper is organized as follows: section 1 introduces the $k$ factor model and discusses its relationship to the Arbitrage Pricing Theory (APT) and Capital Asset Pricing Models (CAPM); section 2 analyses the relation between the two models and the statistical models, while section 3 reviews maximum likelihood inference in reduced rank regression models. Section 4 reports empirical application of these methods to stock returns from the Milan Stock Exchange, comparing them with corresponding inferences based on factor analysis; section 5 contains some concluding remarks.

\section{2 - Latent factors and asset pricing}

The empirical analysis of financial markets makes often use of linear models in the factors; several theories are in fact nested within such models. Two notable models in this class are the (intertemporal) Capital Asset Pricing Models (CAPM) and the Arbitrage Pricing Theory (APT).

Both the static CAPM and the Consumption CAPM hold that one factor (the market portfolio or the growth rate of consumption) is sufficient in explaining asset returns variation. The Intertemporal CAPM, on the whole, is consistent with the view of asset returns being functions of state variables; optimal portfolio selection implies that prices (returns) depend on the covariance of such variables with each marginal utility, which could be approximated by a linear function of the state variables, thus leading to a linear-in-the-factors model.

The APT assumes that the expected asset returns are generated by a factor model:

$$
E_{i}\left(r_{i t} \mid f_{1 t}, \ldots, f_{k l}\right)=E_{i}\left(r_{i t}\right)+\sum_{h=1}^{k} \alpha_{i h} f_{h t} \quad(i=1,2, \ldots n)
$$

where $r_{i}$ is the $i$-th asset return at time $t, E_{i}(\cdot)$ denotes expectations conditional on information known at time $t-1, f_{h t}$ is the $h$-th factor at time $t$ for which $E_{l}\left(f_{h l}\right)=0$; the $\alpha_{i j}$ parameters are here assumed to be constant through time. In matrix form
(1)

$$
E_{t}\left(r_{t} \mid f_{t}\right)=E_{t}\left(r_{t}\right)+\alpha f_{t}
$$
where $r_{t} \equiv\left(r_{1 b}, \ldots, r_{n t}\right)^{\prime}, f_{t} \equiv\left(f_{11}, \ldots, f_{k t}\right)^{\prime}, \alpha=\left[\alpha_{i h}\right], i=1, . ., n, h=1, \ldots, k$.

Ruling out arbitrage opportunities under certain assumptions implies $^{3}$

$$
E_{i}\left(r_{i t}\right)=\lambda_{\alpha}+\sum_{j=1}^{k} \alpha_{i j} \lambda_{j i} \text { or }
$$

$$
E_{i}\left(r_{l}\right)=i_{n} \lambda_{\alpha r}+\alpha \lambda_{r}
$$

where $\lambda_{a}$ is usually interpreted as the return on the risk-free asset, $\lambda_{r} \equiv\left(\lambda_{1}, \ldots, \lambda_{k s}\right)^{\prime}$ and $i_{n} \equiv(1, \ldots, 1)^{\prime}$ is a unit vector of dimension $n$.

The conditional CAPM ${ }^{4}$ could be interpreted as a special case of (1) (2), obtained by setting $k=1$ and

$$
f_{1 t}=r_{m s}-\lambda_{0 s}-\lambda_{1 t}
$$

where $r_{m}$ is the return on the market portfolio. While the existence of only one factor is consistent with the CAPM, it is possible that the single factor does not coincide with the market return. Nevertheless evidence of a single factor, in case of an observable risk free asset $\lambda_{0,}=r_{f}$, and of two factors, in the case of an unobservable portfolio $r_{0}$ orthogonal to the market portfolio $\lambda_{o x}=E_{i}\left(r_{0}\right)$, can be interpreted as consistent with the CAPM; on the other hand more than 2 factors are usually interpreted as evidence in favour of the APT. Both the CAPM and APT can be nested within the unifying equation (1), as shown by Wei $(1988)^{5}$, interpreting the last factor as the market return.

Two important issues must be addressed in specifying the economic implications of both financial models: the time constancy of $\lambda_{r}$ and the observability of $f_{t}$. We will always take the risk-free rate $\lambda_{\alpha}$ to be varying through time. In the next section we will show how the different choices with respect to these two basic questions hint at different statistical models.

Empirical applications on the APT have either focused on extracting the latent factors by factor analysis techniques, without specifying the underlying state variables, or equated the $k$ factor with observable variables on a priori ground, thus obtaining linear regression equations.

3 See Ross(1976), Connor (1984), Wei (1988).

4 See Gibbons and Ferson (1985), Burmeister and McElroy (1991).

5 See also Burmeister and McElroy (1991). 
While the former procedure has been questioned as leading to too many factors ${ }^{6}$, the latter does not provide a test of the number of factors, which can discriminate between alternative models as the APT and CAPM.

This paper follows a different approach to test the two competing financial models. In the next section, in fact, we analyze the two basic statistical models usually considered in applied work: the factor analysis model and the regression model; it is found that the type of restrictions (1) and (2) lead in both cases to reduced rank regression models which thus represents an unifying scheme. The reduced rank structure allows the researcher to test for the number of latent factors in asset returns with or without macroeconomic indicators. Moreover it allows to asses the relation between financial markets and each economic indicator within the same model, i.e. characterized by the same number of factors.

\section{3 - The statistical models}

In this section we will assume that the implications of financial models of the previous section on returns can be represented in the form (4)

$$
E_{t}\left(r_{t} \mid f_{t}\right)=\alpha\left(f_{t}+\lambda_{t}\right)
$$

If the risk free return $\lambda_{0,}$ is observed, $r_{t}$ can be set equal to the vector of excess returns of the $n$ assets with respect to the risk free rate $\lambda_{\alpha}$. If there is no risk free asset, then the last vector in parenthesis on the right-hand-side of (4) can be expanded to contain the extra component $E_{s}\left(r_{\alpha}\right)=\lambda_{\alpha}$.

The two major specification problems in (4) concern the observability of $f_{t}$, and the time constancy of $\lambda_{t}$. Let us first consider the latter; if there is no time variation in $\lambda_{1}=\lambda$ eq. (4) becomes

$$
E_{t}\left(r_{t} \mid f_{t}\right)=\mu+\alpha f_{t}
$$$$
\text { (5) }
$$

$$
\mu=\alpha \lambda
$$

Sometimes it does not seem reasonable to expect $\lambda_{r}$ to be constant through time. From eq. (2) one has $\alpha \lambda_{t}=E_{t}\left(r_{t}\right)$, which shows that, as $\alpha$ is a constant matrix of parameters, $\lambda_{r}$ can be in general a function of the information set at time $t-1$. The simplest assumption in this respect is

6 See Conway and Reinganum (1988) and reference therein.

$$
\lambda_{\mathrm{s}}=\delta g_{\mathrm{s}}
$$

where the $s \times 1$ vector $g_{t}$ contains observable variables in $t-1, s>k^{7}$. Substituing one has

(7)

or, considering eq. (4),

$$
E_{r}\left(r_{1}\right)=\alpha \delta g_{i}
$$

where

$$
E_{t}\left(r_{t} \mid f_{t}\right)=\phi g_{t}+\alpha f_{t}
$$

$$
\phi=\alpha \delta
$$

Equations (5) and (8) represent two possible formulations of the financial models on which our analysis will be focused: the former relates to the case of time-constant $\lambda_{t}$, while the latter represents the chosen specification in the case of time-varying $\lambda_{t}$.

The second issue involved in the specification of the model is the one regarding observability of the vector of factors $f_{t}$. If $f_{t}$ is observable, eq. (5) and (8) specify linear regression functions; in other words the relevant reference statistical model in this case is a linear regression model. On the other hand if $f_{t}$ is unobservable the statistical model of reference for eq. (5) and (8) is a factor analysis model, see e.g. Anderson $(1984)^{8}$. Note that interpreting $f_{c}$ as latent factors in (8) specifies a mixed model with both observable and unobservable variables which can still be accommodated in a factor analysis model.

The two reference statistical models, the factor analysis model and the linear regression model, are therefore analyzed in the following subsection under the restrictions (5) (6) and (8) (9).

\section{1 - Implications on the factor analysis model}

The usual formulation of the factor analysis model is the following (10)

$$
r_{t}=\mu+\alpha f_{t}+\varepsilon_{t}
$$

where $\mu$ in a $n \times 1$ vector of constants, and $\varepsilon_{d}$ and $f_{1}$ are i.i.d. normal random variables with zero mean and covariance matrices $E\left(f f_{i}{ }^{\prime}\right)=I_{k}, E\left(f_{l} \varepsilon_{l}{ }^{\prime}\right)=0$,

7 The same sort of assumption is made e.g. in Gibbons and Ferson (1985).

S Some empirical works based on the former model are Chan, Chen and Hsieh 8 Some empirical works based on the former model Warga (1986), Huberman and (1985), Chen, Roll and Ross (1988). Brown (1988) and Costa (1990) for the case of Italy are applications of the latter model. 
$E\left(\varepsilon, \varepsilon_{,}{ }^{\prime}\right)=\Psi=\operatorname{diag}\left(\psi_{1}, \ldots, \Psi_{n}\right)$. It is easy to see that relation (5) can be nested within such a statistical model; eq. (8) seems more at odds with (10); nevertheless one can generalize model $(10)$ in the following way
(11)
$r_{t}=\phi g_{t}+\alpha f_{t}+\varepsilon_{t}$

where

$$
\left(\begin{array}{l|l}
\varepsilon_{t} \\
f_{t}
\end{array} \mid g_{t}\right) \sim N\left(0,\left(\begin{array}{ll}
\Psi & 0 \\
0 & I
\end{array}\right)\right)
$$

It is easy to see that the statistical models (10) and (11) nests both (5) and (8). Note that (5) imposes a restriction on the constant $\mu$ of model (10), and (8) imposes the restriction (9) on the coefficient matrix $\phi$ in (11).

Consider now the partition $r_{t}=\left(r_{1 t}, r_{2 t}^{\prime}\right)^{\prime}$; the following proposition states that in both (10) and (11), the conditional model for $r_{16}$ with respect to $r_{2}$ is a regression model, with matrix of regression coefficients of reduced rank $k$, equal to the number of factors.

\section{Theorem 1}

Let $r_{t} \equiv\left(r^{\prime}, r^{\prime}{ }_{2 t}\right)$ ' be a partition of $r_{t}$ in (10), with $r_{1 s}, r_{2 t}$ of dimension $n_{1} \times 1$ and $n_{2} \times 1$, respectively, $n_{1}, n_{2}>k$. Let (6) also hold and let $\alpha_{1}, \alpha_{2}$ be of full rank $k$. Then the model of $r_{1 t}$ conditional on $r_{2 t}$ is normal with

$$
E\left(r_{1 t} \mid r_{2 t}\right)=\alpha_{1} \beta^{\prime}\left(\begin{array}{c}
r_{2 t} \\
1
\end{array}\right)
$$

and positive definite conditional covariance matrix $\Lambda_{1.2}$. The matrix of regression coefficients $\theta=\alpha_{1} \beta$, has dimensions $n_{1} \times\left(n_{2}+1\right)$ and reduced rank $\mathrm{k}$.

Analogously if (9) holds, for the same partition of $r$, in (11) the model of $r_{1}$ conditional on $r_{2}$ is normal with conditional mean

$$
E\left(r_{1 t} \mid r_{2 t}, g_{t}\right)=\alpha_{1} \beta,\left(\begin{array}{l}
r_{2 t} \\
g_{t}
\end{array}\right)
$$

and positive definite conditional covariance matrix $\Lambda_{1.2}$. Again the matrix $\theta=\alpha_{1} \beta$ ' of dimensions $n_{1} \times\left(n_{2}+s\right)$ has reduced rank $\mathrm{k}$.

Proof See appendix.
The previous proposition asserts that the statistical models (10) and (11) imply conditional regression models for the observables, in which the regression coefficient matrix is of reduced rank $k$ equal to the number of latent factors. This implication will form the basis of the tests presented in this paper.

An immediate consequence of theorem 1 can be obtained marginalizing with respect to $f_{t}$.

Corollary 2

Consider the regression model

$$
r_{\mathrm{t}}=\phi g_{\mathrm{i}}+\varepsilon_{\mathrm{r}}
$$

the model (7) imposes the reduced rank restriction

$$
\text { (9) } \phi=\alpha \delta
$$

Finally let us note that the present approach generalizes the work of Gibbons and Ferson (1985). The two authors, in fact, specify a regression model for $r_{t}$ on information variables $g_{i}$ of the form

$$
E_{r}\left(r_{t}\right)=\delta g_{t}
$$

On the other hand from the economic model $E_{t}\left(r_{t}\right)=\alpha \lambda_{t}$; therefore solving for $\lambda_{t}$ one can show that $\lambda_{t}=\bar{\alpha}^{\prime} \delta g_{1}$, where $\bar{\alpha} \equiv \alpha\left(\alpha^{\prime} \alpha\right)^{-1}$, and thus $E_{t}\left(r_{t}\right)=\alpha \bar{\alpha}, \delta g_{t}$, i.e. the financial models impose a reduced rank structure $\alpha \bar{\alpha} ' \delta$ on $\phi$ in the regression model $r_{t}=\phi g_{t}+\varepsilon_{t}$. Gibbons and Ferson derive analogous restrictions on $\phi$; they express the dependence among the coefficients partitioning $r_{t}$ in $\left(r_{1 b}, r_{2 t}\right)$ where $r_{1 s}$ is $k \times 1$, and representing the $\phi$ coefficients for $r_{2}$ as functions of the ones for $r_{16}$. Note, however, that one can represent the restrictions (9) of $\phi$ in (14) directly as reduced rank restrictions. This allows to obtain the maximum likelihood solution of (14) analytically, see section 3 and does not imply any choice of partition for $r_{t}$.

9 One must note that the parameters of the conditional and of the marginal models will not in general be variation independent, and therefore the analysis of the conditional model will not be as efficient as the analsis of the full model. 


\section{2-Implications on the linear regression model}

If the factors $f_{t}$ are observable, the economic model (5) can be nested within a multivariate regression model of the form

$$
r_{t}=(\alpha, \mu)\left(\begin{array}{c}
f_{t} \\
1
\end{array}\right)+\varepsilon_{t}
$$

where $\varepsilon_{i} \sim$ i.i.d. $N(0, \Omega)$. Analogously (8) can be nested within the enlarged model

$$
r_{t}=(\alpha, \phi)\left(\begin{array}{l}
f_{t} \\
g_{t}
\end{array}\right)+\varepsilon_{t}
$$

The assumption of observability of the factors $f_{t}$ is generally coupled with the identification of the factors with some observable variables, often taken to be macroeconomic indicators. In order to identify such factors, several financial models are usually quoted; Present Value Models $^{10}$, for instance, suggest correlations between stock prices, expected dividend yields and variables connected to the intertemporal rate of substitution; on the other hand Consumption Capital Asset Pricing Models ${ }^{11}$ predict co-movements between stock returns and the growth rate of consumption.

The following proposition illustrates how (5) and (8) restrict the coefficient matrices of the statistical models (16) and (17) to be of reduced rank.

Theorem 3

If $f$, is observable (5) (6) restrict the coefficient matrix in the regression model (16) to be of reduced rank $k$

$$
\text { (18) } \quad r_{i}=\alpha \beta^{\prime}\left(\begin{array}{c}
f_{t} \\
1
\end{array}\right)+\varepsilon_{t}
$$

where $\beta=\left(I_{k}, \lambda\right)^{\prime}$, that is $\theta \equiv \alpha \beta$ ' is of dimensions $n \times(k+1)$ and of reduced rank $\mathbf{k}$.

10 See Shiller (1981) inter alia.

11See e.g. Breeden (1979).
Analogously (8) and (9) restrict the coefficient matrix in model (17) to be of reduced rank $k$

$$
r_{t}=\alpha \beta^{\prime}\left(\begin{array}{l}
f_{t} \\
g_{t}
\end{array}\right)+\varepsilon_{t}
$$

where $\beta=\left(I_{k}, \delta\right)$; the matrix $\theta \equiv \alpha \beta$ ' is of dimensions $n \times(k+s)$ and of reduced rank $\mathbf{k}$.

Proof By substitution.

Observe again that the reduced rank structure of eq. (18) and (19) can be exploited to test for the number of factors $k$. Note that when including variables in $f_{t}$ and $g_{t}$ as regressors in (17) there is no need to specify which variable enters which vector, as the variables are treated symmetrically.

The assumption that the factors $f_{t}$ can in fact be identified with a specified set of observable variables seems a rather restrictive one. Often the econometrician has a certain number of variables as possible candidates for the factors, but no definite single choice. A very simple hypothesis in this respect would be to assume (20)

$$
f_{t}=\tau h_{t}
$$

where $h_{t}$ is a $p \times 1$ vector of observable variables and $\tau$ is a $k \times p$ matrix of coefficients, with $p>k$. In other words the underlying factors $f_{t}$ are linear combinations of observable variables. Note that under assumption (20) the researcher is allowed to proceed in a general to specific fashion in the search for the set of indicators. It is simple to show that under assumption (20) theorem 3 is still valid; more precisely

Corollary 4

Under the assumptions of theorem 3 and (20), eq. (18) and (19) are still valid when substituting $h_{i}$ for $f_{t}$, that is

$$
r_{t}=\alpha \beta^{\prime}\left(\begin{array}{c}
h_{t} \\
1
\end{array}\right)+\varepsilon_{t}
$$

where in this case $\beta=(\tau, \lambda)^{\prime}, \theta=\alpha \beta$, is of dimensions $n \times(p+1)$ and of reduced rank $\mathrm{k}$; analogously

$$
r_{t}=\alpha \beta^{\prime}\left(\begin{array}{l}
h_{t} \\
g_{t}
\end{array}\right)+\varepsilon_{t}
$$

where $\beta^{\prime}=(\tau, \delta), \boldsymbol{\theta}=\alpha \beta^{\prime}$ is $n \times(p+s)$ and reduced rank $\mathbf{k}$. Proof By substitution. 
An economically interesting question of own right concerns the relevance of each economic indicator in $h_{i}$; note that if a variable is incorrectly included in $h_{b}$, the corresponding column of $\tau$ is actuaily a zero column. Hypothesis of the type $R$ ' $\beta=0$ therefore turn out to be of interest, which can also be formulated as

\section{(21)} $\beta=H \psi$

In the section 4 we will therefore analyze hypotheses of the form (21) as specification tests with respect to (20).

\section{3 - Asset returns and macroeconomic dynamics}

While both theory and empirical evidence suggest that asset returns are hardly serially correlated, macroeconomic variables exhibit strong temporal dependence. Often a vector autoregressive data generating process is assumed as a possible model for the dynamics of macroeconomic indicators; such a process nests, for instance, a structural dynamic system unrestricted reduced form. In this subsection we try to accommodate dynamics for the macroeconomic indicators specifyed in $g_{t}$ and $h_{t}$. Let $m_{t}$ be a $l \times 1$ vector of macroeconomic variables generated by the following model
(22)
$A(L) m_{t}=\mu_{m}+\gamma f_{t}+\xi_{t}$

where $A(L) \equiv I-A_{1} L-A_{2} L^{2}-\ldots-A_{q} L^{q}$ is a finite matrix polynomial in the lag operator $L, \mu_{m}$ is a vector of constants, $f_{t}$ and $\xi_{t}$ are assumed i.i.d. normal uncorrelated random vectors $E\left(\xi_{t} \xi_{t}{ }^{\prime}\right)=\Sigma=\operatorname{diag}\left(\sigma_{1}^{2}, \ldots, \sigma_{l}^{2}\right)$ and $E\left(\xi_{1} \varepsilon_{\mathrm{r}}{ }^{\prime}\right)=0$. It is well known that the stationarity of the system is linked to the roots of the determinantal equation $|A(z)|=0$, given the assumption that both $f_{t}$ and $\xi_{t}$ are serially uncorrelated. Note that we are now back to the assumption that $f_{t}$ is unobservable. In the empirical application we assume that such roots are all outside the unit circle, i.e. that the system is stationary. Nevertheless specification (22) is general enough to analyze non stationary - and possibly cointegrated - systems, thus providing an interesting area of future research.

Theorem 1 can be applied to (10), (22) obtaining the following proposition, a proof of which is reported in the appendix.
Corollary 5

In the statistical models (10), (22) or (11), (22) the conditional models of $\left(r_{t} \mid m_{t}\right)$ and $\left(r_{t} \mid m_{t}, g_{t}\right)$ are of the type (12) and (13), with $r_{t}$ in place of $r_{1 t}$ and $\underline{m}_{t}=\left(m_{t}^{\prime}, m_{t-1}, \ldots, m_{t-q}\right)^{\prime}$ in place of $r_{21}$, i.e.

$$
E_{t}\left(r_{t} \mid m_{t}\right)=\alpha \beta^{\prime} m_{t} \quad E_{t}\left(r_{t} \mid m_{t}, g_{t}\right)=\alpha \beta^{\prime}\left(\begin{array}{c}
m_{t} \\
g_{t}
\end{array}\right)
$$

Therefore the rank of the regression matrix of returns on current and lagged values of macroeconomic indicators is also equal to the number $k$ of underlying factors; a reduced rank regression of these variables can then also serve as a test of the theories.

\section{4 - Inference in reduced rank regression models}

The reduced rank model has been analyzed extensively in the statistical literature (Anderson 1951,76, Khatri 1976, Izemnan 1975, Tso 1981, Johansen 1989, 91), while it has not received much emphasis in the financial literature. The maximum likelihood problem in the reduced rank regression model is solved by calculating the canonical variates between the independent and dependent variables, after conditioning on the other variables $w_{t}$ at the outset. The analytic solution provides the means of constructing simple likelihood ratio tests of the various restrictions; the rank constraint makes it possible to test for the number of factors, while other restrictions can be tested conditionally on the number of underlying factors. Several tests and maximum likelihood estimators are reviewed in the following subsections.

Consider the following regression model

$$
\text { (24) } y_{t}=\underset{n \times 1}{\alpha} \underset{n \times k}{\alpha} \underset{k \times p}{\beta} \underset{p \times 1}{x_{i}}+\underset{n \times s}{\delta} \underset{\substack{1 \\ s \times 1}}{w_{t}}+\underset{n \times 1}{\varepsilon_{1}} \quad t=1, \ldots, T
$$

where $\varepsilon_{t}$ is a vector gaussian white noise, $\varepsilon_{t} \mid x_{t}, w_{t} \sim N(0, \Omega)$. It is easy to see that the models of the previuos section (see (12) (13) (14) (18) (19)) are nested within (24).

The identification problem of $\alpha$ and $\beta$ is evident in the reduced rank model (24): given a pair of $\alpha$ and $\beta$ matrices, all the possible pairs $\alpha^{*}=\alpha \xi^{,-1}$ and $\beta^{*}=\beta \xi$ are observationally equivalent, where $\xi$ is any non-singular matrix, as $\alpha \beta^{\prime}$ and $\alpha^{*} \beta^{*}$ ' are equal. Non-the-less the column spaces of both $\alpha$ and $\beta$ can be identified, that is up to a normalizing rotation ${ }^{12}$.

12 Which consists in a choice of $\xi$, and thus is shared by both $\alpha$ and $\beta$. 
Such a normalization can be chosen so as to either obtain $\beta=\left(I_{k}, \beta_{o}{ }^{\prime}\right)$ ' or $\beta^{\prime} S_{x x} \beta=I_{k}$, where $S_{x x}$, is defined in the following. Both normalizations impose $k^{2}$ identification restrictions; the number of parameters contained in $\alpha \beta^{\prime}$ is therefore equal to $n k+k p-k^{2}$.

The maximum likelihood problem in reduced rank regressions is solved analytically by the canonical correlation problem between $y_{t}$ and $x_{t}$ conditional on $w_{t}$, as shown e.g. in Anderson (1951) and Tso (1981). Consider the log likelihood of model (24)

$$
\ln L(\alpha, \beta, \delta, \Omega)=-\frac{n T}{2} \ln (2 \pi)-\frac{T}{2} \ln |\Omega|-\frac{1}{2} \sum_{t} \varepsilon_{t}{ }^{\prime} \Omega^{-1} \varepsilon_{t}
$$

The likelihood function can be concentrated with respect to $\delta$ simply regressing $y_{t}$ and $x_{t}$ on $w_{t}$ and considering the residuals

$$
R_{y t}=y_{t}-M_{y w} M_{w w}^{-1} w_{t} \quad R_{x t}=x_{t}-M_{x w} M_{w w}^{-1} w_{t}
$$

where

$$
M_{a, b}=\frac{1}{T} \sum_{i=1}^{T} a_{t} b_{t} \quad a, b=y, x, w
$$

The concentrated likelihood function is therefore

$$
\ln L(\alpha, \beta, \Omega)=\mathrm{const}-\frac{T}{2} \ln |\Omega|-\frac{1}{2} \sum_{t=1}^{T}\left(R_{y t}-\alpha \beta^{\prime} R_{x t}\right)^{\prime} \Omega^{-1}\left(R_{y t}-\alpha \beta^{\prime} R_{x t}\right)
$$

For fixed $\beta$ one can maximize $\ln L$ with respect to $\alpha$ regressing $R_{y t}$ on $\beta ' R_{x z}$ obtaining

where

$$
\hat{\alpha}(\beta)=S_{y x} \beta\left(\beta^{\prime} S_{x x} \beta\right)^{-1} \quad \hat{\Omega}(\beta)=S_{y y}-S_{y x} \beta\left(\beta^{\prime} S_{x x} \beta\right)^{-1} \beta^{\prime} S_{x y}
$$

$$
S_{a, b}=\frac{1}{T} \sum_{t=1}^{T} R_{a r} R_{b x}^{\prime}=M_{a b . w} \quad a, b=x, y
$$

The likelihood function concentrated with respect to $\alpha$ and $\delta$ is

$$
\ln L(\beta)=\text { const }-\frac{T}{2} \ln \left|S_{y y}-S_{y x} \beta\left(\beta^{\prime} S_{x x} \beta\right)^{-1} \beta ' S_{x y}\right|
$$

which can be maximized by minimizing the determinant on the right hand side. From the properties of determinants
(25)

$$
\left|S_{y y}-S_{y x} \beta\left(\beta^{\prime} S_{x x} \beta\right)^{-1} \beta^{\prime} S_{x y}\right|=\frac{\left|s_{y}\right|\left|\beta^{\prime}\left(s_{x x}-S_{x y} s_{y y}^{-1} S_{y x}\right) \beta\right|}{\left|\beta^{\prime} S_{x x} \beta\right|}
$$

Minimizing (25) is tantamount to solving the canonical correlation problem between $y$ and $x$ conditional on $w$, i.e. it corresponds to the eigenvalue problem ${ }^{13}$

$$
\left|u S_{x x}-S_{x y} S_{y y}^{-1} S_{y x}\right|=0
$$

where the ordered eigenvalues $u_{1} \geq u_{2} \geq \ldots \geq u_{p}$ are the squared canonical correlations ${ }^{14}$. The maximum likelihood estimator of $\beta$ is given by the $k$ eigenvectors associated with the first $k$ canonical correlations.

The maximized likelihood function is thus proportional to, for given $k$,

$$
\ln L_{\max }(k)=\text { const }-\frac{T}{2}\left|S_{y y}\right|-\frac{T}{2} \sum_{i=1}^{k} \ln \left(1-u_{i}\right)
$$

The likelihood ratio test of the hypothesis $k \leq d$ within the unrestricted model $k=p$ is given by ${ }^{15}$

$$
Q_{1}^{*}(d)=-2 \ln \left(\frac{L_{\max }(k \leq d)}{L_{\max }(k=p)}\right)=-T \sum_{i=d+1}^{p} \ln \left(1-u_{i}\right) \quad d=0,1, \ldots, p-1
$$

while the likelihood ratio tests of the hypothesis $k \leq d$ versus $k=d+1$ is just

$$
Q_{2}^{*}(d)=-2 \ln \left(\frac{L_{\max }(k \leq d)}{L_{\max }(k \leq d+1)}\right)=-T \ln \left(1-u_{d+1}\right) \quad d=0,1, \ldots, p-1
$$

The test statistics $Q_{1}^{*}, Q_{2}^{*}$ are asymptotically distributed as $\chi^{2}$ under general hypothesis ${ }^{16}$, the degrees of freedom being equal to the number of restrictions, $v_{1}=(n-d)(p-d)$ and $v_{2}=(n-d)+(p-d)-1$ respectively. Applying Bartlett corrections one obtains the statistics

$$
\begin{aligned}
& Q_{1}(d)=-\left(T-\frac{1}{2}(n+p+3)\right) \sum_{i=d+1}^{p} \ln \left(1-u_{i}\right) \\
& Q_{2}(d)=-\left(T-\frac{1}{2}(n+p+3)\right) \ln \left(1-u_{d+1}\right) \quad d=0,1, \ldots, p-1
\end{aligned}
$$

13 See appendix.

14 See Tso (1981), pg. 187.

15 Here we assume that $p<n$. In case $p>n$ one just needs to intechange $p$ and $n$, as the rank of $\alpha \beta^{\prime}$ is at most equal to the minimum between the number of rows and the number of columns.

16 See e.g. Anderson (1984); these hypothesis do not cover the nonstationary case, as in tests for cointegration, see Johansen $(1989,1991)$. 
which are again $\chi^{2}$ distributed with $v_{1}$ and $v_{2}$ degrees of freedom respectively.

The $Q_{2}(d)$ statistics provide a sequence of conditional tests. On the other hand an alternative consistent testing procedure is obtained considering the sequence of test statistics $Q_{1}(0), Q_{1}(1), Q_{1}(2) Q_{1}(p-1)$ and choosing for $k$ the first value for which $Q_{1}(k)$ is not significant at the specified significance level ${ }^{17}$. Asymptotically an incorrect smaller dimension $k$ would never be chosen, while the probability of choosing the correct value is at least equal to $1-\alpha$. In the following we rely on the latter procedure.

\section{1 - Specification tests}

Once the rank of $\theta=\alpha \beta$, has been selected, it would be interesting to test linear restrictions on $\beta$, as e.g. exclusion restrictions in equation ( $9^{\prime}$ ), which would correspond to the irrelevance of some of the chosen independent variable with respect to the factor model. Consider the following linear hypothesis in explicit form

$$
\text { (27) }
$$

$$
\beta=H \psi
$$

which is equivalent to the implicit form

$$
R^{\prime} \beta=0
$$

where $H$ is a known matrix of dimensions $(p \times q), R=H_{\perp}$, and indicates $_{\perp}$ the orthogonal complement of a matrix, that is $H_{\perp}: H_{\perp}{ }^{\prime} H=0,\left(H, H_{1}\right)$ span $\Re^{p}$.

The exclusion hypothesis of one of the variables included in $x_{t}$ from the model , for example, corresponds to $R=(1,0, \ldots 0)^{\prime}$ the following equivalent formulations, for which it is easy to verify the orthogonality of the given matrices

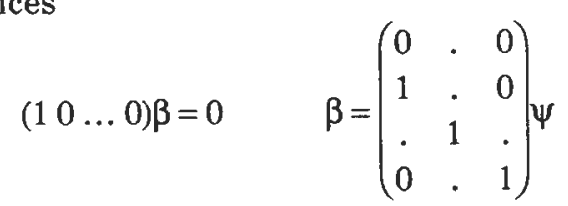

17 See e.g. Pantula (1989) and reference therein.
Under the restrictions (27) the likelihood function can be maximized just along the lines of the previous section, see Johansen $(1989,1991)$; the relevant eigenvalue problem becomes

$$
\mid u^{*} S_{x x}^{*}-S_{x y}^{*} S_{y y}^{-1} S_{y x}^{*} \neq 0
$$

where $S_{x x}^{*}=H^{\prime} S_{x x} H, S_{x y}^{*}=H^{\prime} S_{x y}$, with $u_{1}^{*} \geq u_{2}^{*} \geq \ldots \geq u_{q}^{*}$ ordered eigenvales; the maximum likelihood estimator of $\psi$ is given by the first $k$ eigenvectors. The maximized likelihood function is

$$
\ln L_{\max }(k, H)=\text { const }-\frac{T}{2}\left|S_{y y}\right|-\frac{T}{2} \sum_{i=1}^{k} \ln \left(1-u_{i}^{\prime}\right)
$$

and the likelihood ratio test of the restrictions (27) leads to the statistic

$$
{ }_{H} Q_{3}^{*}(k)=-T \sum_{i=1}^{k} \ln \left(1-u_{i}\right)+T \sum_{i=1}^{k} \ln \left(1-u_{i}^{*}\right)
$$

which is again asymptotically $\chi^{2}$ distributed with $v_{3}=(p-q) k$ degrees of freedom.

\section{2 - A test based on the constant term}

Both the CAPM and the APT specification restrict the vector of constant to lie in the column space of $\alpha$, that is

$$
\mu=\alpha \lambda
$$

Such a restriction appears in (12) and it is in fact testable. The unrestricted model with no constraints on the constant can be estimated as in the section 3.1 simply setting $w_{t}=1$. Let the maximized likelihood function be denoted by

$$
\ln L_{\max }(k)=\text { const }-\frac{T}{2}\left|\tilde{S}_{y y}\right|-\frac{T}{2} \sum_{i=1}^{k} \ln \left(1-\tilde{u}_{i}\right)
$$

The likelihood ratio test of the restrictions (6) is therefore based on the statistic

$$
Q_{4}^{*}(k)=-T \sum_{i=1}^{k} \ln \left(1-\tilde{u}_{i}\right)+T \sum_{i=1}^{k} \ln \left(1-u_{i}\right)-T\left|\tilde{S}_{y y}\right|+T\left|S_{y y}\right|
$$

which is asymptotically $\chi^{2}$ distributed with $n-k$ degrees of freedom. The rejection of the restrictions (6) would imply e.g. "preferred habitat" effects in the market. 


\section{5 - An empirical application}

In the empirical analysis we considered 29 monthly asset returns $\mathrm{s}^{18}$ from the Milan Stock Exchange, from the period January 1972 December 1989, the choice of monthly returns being linked to the availability of macroeconomic indicators only on a monthly basis. Previous studies indicate that the Italian Stock Market was too thin before the 1980s (see Baccolini and Lusignani, 1991); on the other hand homogeneous macroeconomic time series are available only for the last decade; we therefore selected the sample January 1980 to December 1989 as our sample period.

In the specification of the models of section 2 one can easily meet a dimensionality problem, as many variables are considered simultaneously $\left(r_{t}, g_{t}, h_{t}, m_{t}\right)$. Although a general to simple approach would suggest to consider all variables at one time, the empirical ratio of the number of parameters to degrees of freedom forced us to break down the analysis.

We therefore first analyzed the autocorrelation structure of returns and checked for the presence of deterministic calendar effects. Table 1 reports the first 12 autocorrelations of the assets returns for the period February 1972 - December 1989: 32 over the 348 calculated autocorrelations (about $9 \%$ ) are outside the interval $\pm 2 / \sqrt{n}$, thus indicating a slightly greater rejection rate than expected. Since 9 significant autocorrelations were detected at lag 12 in the univariate analysis, a multiple regression of $r_{\text {, on }} r_{t-12}$ and a constant and 11 seasonal dummies was estimated in order to check for a seasonal multivariate dependence at lag 12. The relevant statistics reported in table 2 do not indicate any significant effect. Also restricting the model to deterministic seasonal factors, no apparent significant deterministic seasonal components were detected, see table $3^{19}$.

18 The analyzed returns, measured in percentage points, are the following common stocks: Eridania, Alleanza, Generali, RAS, SAI, Toro, Comit, Banco di Roma, Medibanca, Burgo, Italcementi, Montedison, Pirelli, Rinascente, Alitalia Priv., Italcable, SIP, Acqua Marcia, IFI Priv., Pirelli \& C., SME, STET, Risanamento Napoli, Fiat, Olivetti, Falck, Cantoni, Ciga, Pacchetti.

19 Even if the value of June is not strictly insignificant; similar results were obtained in Paruolo and Costa (1989).
We therefore considered model (10) (12) as a tentative first model for returns. Exploratory factor analysis applied on the given 29 returns indicated 6 or 7 factors according to the $1 \%$ or $5 \%$ critical values for the likelihood ratio criterion, see table 4 . The reduced rank regression model (12) was fitted with $n_{1}=15$ and $n_{2}=14$; the results indicate, instead, only 1 factors, see table 5 . The reduced rank regression approach seem therefore to uncover much fewer significant factors.

Turning to models with macroeconomic variables one needs to specify $g_{t}, h_{t}$ or $m_{t}$, see (8), (20) and (22). The following macroeconomic indicators were considered in the empirical analysis: the inflation rate (INFL), the logarithm of the industrial production index (PI), the interest rates on the 3 month Treasury Bills (BOT), the interest rates on government bonds (BTP), the logarithm of the US dollar - Italian lira exchange rate (USD), the logarithm of the deutsche mark - lira exchange rate (DM), the oil price index (OIL), the Milan Stock Exchange index (MIB), the discount rate changes (DTUS), the logarithm of money (M2). For the choice of the macroeconomic indicators see, e.g., Hamao, 1986. The monthly time series of some of these variables in the decade 1980-1989 are shown in figures 1,2 and 3.

The difference between BOT and BTP was also included in place of the latter, in accordance with the notion of interest rates being cointegrated with a stable differential ${ }^{20}$. The inclusion of such a variable allows us to evaluate the influence of the term structure of interest rates on assets returns. Both the graphical analysis and tests for unit roots suggest that most the macroeconomic variables are non-stationary, see table 6 . In order to obtain stationary variables we considered first differences of the variables.

The industrial production index and M2 show, moreover, a strong seasonal pattern; these series were filtered fitting a regression model in first differences on seasonal dummies, thus retaining interpretability of the seasonally adjusted series, even though the hypotheses of constant seasonal factors does not seem to be a very good approximation for M2. Figures 2 and 3 show both the seasonally unadjusted and the seasonally adjusted series.

20 All possible other cointegrating relations have been neglected in the empirical application. 
The analysis of model (18') or (19') reveals that the vectors of returns $r_{t}$ and the vector of indicators $h_{t}$ should present the same autocorrelation structure as the variables are linearly related. As indicated by the previous analysis, $r_{t}$ is hardly autocorrelated, while there is no reason to believe the set of macro-indicators to be serially uncorrelated; we therefore projected the above variables on a constant and own lags using fitted residuals as our choice of $h_{i}$. The results of the reduced rank regression of $r_{t}$ on $h_{t}$ and a constant are reported in table 7. Basing inference on the number of factors on the statistics $Q_{1}(j)$, as illustrated in section 3 , one is lead to choose 3 relevant factors at the $1 \%$ significance level or 4 at the $5 \%$ significance level.

In this model it is possible to investigate the relevance of each macroeconomic indicator within the chosen specification on the basis of the ${ }_{H} Q_{3}^{*}(k)$ statistics. Table 8 reports the specification tests of type (21) with $H$ being set to the identity matrix without a column corresponding to the chosen variable. Such a choice corresponds to the exclusion of the variable from the vector $h_{t}$

The results indicate that the inflation rate (INFL), the exchange rates (DM, USD) and the oil price (OIL) are not relevant in the identification of latent factors ${ }^{21}$. On the contrary the Milan stock exchange index (MIB), the 3 months Treasury Bills interest rate (DBOT) and money (DM2) appear to be relevant; moreover the interest rate differential BOT-BTP seems to be only marginally significant. Both the number of factors and the significance of additional macroeconomic indicators besides the stock market index favour an APT model for the italian stock returns.

Macroeconomic dynamics can be explicitly incorporated into the model considering (22) and corollary 5. Table 9 reports the results on the number of factors in such a model; the $Q_{1}$ statistics suggests 3 to 4 factors, which is still less than the number indicated by factor analysis and is in accordance with the previous model. On the whole the reduced rank regression approach seems to lead to a smaller number of significant factors.

21 The above results must be taken with care, as each hypotheses is tested independently against the full model.

\section{6 - Conclusions}

In this paper several models are derived from a $k$ factor model for asset returns and a linear specification for the relationship between latent factors and macroeconomic indicators. All the derived models can be cast in the reduced rank regression model. In such a model the maximum likelihood estimator for the parameters of interest is found by canonical correlation analysis; several tests for the number of latent market factors can be based on the likelihood ratio principle, as all the models can be easily estimated. The search for the number of factors underlying returns structure and the consequent inference on alternative assets price theories, as the APT and the CAPM, is straightforward as each one implies a different number $k$ of the latent factors.

The empirical application of some of the inferential procedures outlined in the paper on a sample of monthly returns of assets from Milan Stock Exchange has lead to interesting results. While the application of usual factor analysis procedures indicates 6 to 7 latent factors, reduced rank regression techniques indicate fewer significant factors. Among the included macroeconomic indicators only the inflation rate and the exchange rates are not relevant in the identification of the latent factors. The results are, on the whole, consistent with an APT price generation scheme. 


\section{References}

T.W. Anderson (1951), Estimating linear restrictions on regression coefficients for multivariate normal distributions, Annals of Mathematical Statistics.

T.W. Anderson (1976), Estimation of linear Functional Relationships, Journal of the Royal Statistical Society, B, 38, 1-36.

T.W. Anderson (1984), An Introduction to Multivariate Statistical Analysis, New York, Wiley.

R. Baccolini, G. Lusignani (1991), The Milan stock exchange 1991, Prometeia, Bologna.

D.T. Breeden (1979), An Intertemporal Asset Pricing Model with Stochastic Consumption and Investment Opportunities, Journal of Financial Economics, 7, 265-296.

D. Brown (1988), The Implications of Nonmarketable Income for Model of Asset Pricing, Journal of Financial Economics, 867-880.

E. Burmeister, M. McElroy (1991), The residual Market Factor, the APT, and Mean-Variance Efficiency, Review of Quantitative Finance and Accounting, 1, 27-49.

KC. Chan, N. Chen, D. Hsieh (1985), An exploratory Investigation of the Firm Size Effect, Journal of Financial Economics, 14, 451-471.

N. Chen, R. Roll, S. Ross (1986), Economic Forces and the Stock Market, Journal of Business, 59, 383-403.

G. Connor (1984), A unified Beta pricing Theory, Journal of Economic Theory, 34, 13-31.

D. A. Conway, M.R. Reinganum (1988), Stable Factors in Security Returns: Identification Using Cross-Validation, Journal of Business \& Economic Statistics, vol 6, 1-15.

M. Costa (1990), Applicazioni finanziarie di tecniche fattoriali: un'analisi di sensitività, Atti del convegno "Errori nelle variabili e variabili latenti in modelli strutturali stocastici", Firenze, 6-7 dicembre 1990.

W. Ferson (1990), Are the Latent Variables in Time-Varying Expected Returns Compensation for Consumption Risk?, Journal of Finance, XLV, pp. 397-429

M. Gibbons, W. Ferson (1985), Tests of Asset Pricing Models with Changing Expectations and an Unobservable Market Portfolio, Journal of Financial Economics, 14, 217-236.

Y. Hamao (1988), An Empirical Examination of the Arbitrage Pricing Theory, Japan and the World Economy, 1, 45-61.
G. Huberman, S. Kandel (1987), Mean Variance Spanning, Journal of Finance, 42, 873-888.

A.J. Izemnan (1975), Reduced Rank Regression for the Multivariate Linear Model, Journal of Multivariate Analysis, 5, 248-264.

S. Johansen (1989), Likelihood based inference of cointegration: Theory and applications, Lecture notes, Bagni di Lucca 1989.

S. Johansen (1991), Estimation and Hypothesis testing of cointegration vectors in Gaussian Vector Autoregressive Models, Econometrica, 59, pp. 1551-1580.

C.G. Khatri (1976), A Theorem on Least Squares Multivariate Linear Regression, Journal of the American Statistical Association, 62, 1494-1495.

S.G. Pantula (1989), Testing for unit roots in time series data, Econometric Theory, 5, 256-271.

P. Paruolo, M. Costa (1989), Informazione e Capital Asset Pricing Models: una verifica empirica su dati italiani, Statistica, XLIX, 3, 427-439.

S.A. Ross (1976), The Arbitrage Theory of Capital Asset Pricing, Journal of Economic Theory, 13, 341-360.

R.J. Sweenery, A.D. Warga (1986), The pricing of Interest Rate Risk, Journal of Finance, 41, 393-410.

R.J. Shiller (1981), Do Stock Prices Move Too Much to be Justified by Subsequent Changes in Dividends? , American Economic Review, 71, 421-436.

M.K.S. Tso (1981), Reduced Rank Regression and Canonical Analysis, Journal of the Royal Statistical Society, Serie B, 43, 183-189.

K. Wei (1988), An Asset Pricing Theory Unifying the CAPM and APT, The Journal of Finance, 881-882. 


\section{Appendix}

\section{Proof of theorem 1}

Consider the distribution of $r_{t}$ in model (10):

$$
r_{i}=\left(\begin{array}{l}
r_{11} \\
r_{2}
\end{array}\right) \sim N\left(\left(\begin{array}{l}
\mu_{1} \\
\mu_{2}
\end{array}\right),\left(\begin{array}{cc}
\alpha_{1} \alpha_{1}{ }^{\prime}+\Psi_{1} & \alpha_{1} \alpha_{2}^{\prime} \\
\alpha_{2} \alpha_{1}^{\prime} & \alpha_{2} \alpha_{2}^{\prime}+\Psi_{2}
\end{array}\right)\right)
$$

The conditional distribution $r_{1 t} \mid r_{2 t}$ is normal with conditional mean

$$
\begin{aligned}
E\left(r_{1 t} \mid r_{2 t}\right) & =\mu_{1}+\alpha_{1} \alpha_{2}{ }^{\prime}\left(\alpha_{2} \alpha_{2}{ }^{\prime}+\Psi_{2}\right)^{-1}\left(r_{2 t}-\mu_{2}\right) \\
& =\mu_{1}{ }^{*}+\alpha_{1} \beta_{1}{ }^{\prime} r_{2 t}
\end{aligned}
$$

where $\beta_{1}{ }^{\prime} \equiv \alpha_{2}{ }^{\prime}\left(\alpha_{2} \alpha_{2}{ }^{\prime}+\Psi_{2}\right)^{-1}$, and conditional variance

$$
\Lambda_{1.2} \equiv \operatorname{Var}\left(r_{16} \mid r_{2}\right)=\alpha_{1} \alpha_{1}^{\prime}+\Psi_{1}-\alpha_{1} \alpha_{2}^{\prime}\left(\alpha_{2} \alpha_{2}{ }^{\prime}+\Psi_{2}\right)^{-1} \alpha_{2} \alpha_{1}{ }^{\prime}
$$

Under (6) $\mu_{1}=\alpha_{1} \lambda, \mu_{2}=\alpha_{2} \lambda$, and therefore

$$
\begin{aligned}
E\left(r_{1 t} \mid r_{2 t}\right) & =\alpha_{1} \lambda+\alpha_{1} \alpha_{2}{ }^{\prime}\left(\alpha_{2} \alpha_{2}{ }^{\prime}+\Psi_{2}\right)^{-1}\left(r_{2 t}-\alpha_{2} \lambda\right)= \\
& =\alpha_{1} \beta_{1}{ }^{\prime} r_{2 t}+\alpha_{1}\left(I_{k}-\beta_{1}{ }^{\prime} \alpha_{2}\right) \lambda=\alpha_{1} \beta_{1}{ }^{\prime} r_{2 t}+\alpha_{1} \beta_{2}= \\
& =\alpha_{1}\left(\beta_{1}{ }^{\prime}, \beta_{2}\right)\left(\begin{array}{c}
r_{2 t} \\
1
\end{array}\right)=\alpha_{1} \beta^{\prime}\left(\begin{array}{c}
r_{2 t} \\
1
\end{array}\right)
\end{aligned}
$$

where $\beta_{2} \equiv\left(I_{k}-\beta_{1}{ }^{\prime} \alpha_{2}\right) \lambda$ is a $k \times 1$ vector.

Analogously consider the distribution of $r_{t}$ conditional on $g_{t}$

$$
\left.\left(\begin{array}{l}
r_{1 t} \\
r_{2}
\end{array}\right)\right) g_{1} \sim N\left(\left(\begin{array}{l}
\phi_{1} \\
\phi_{2}
\end{array}\right) g_{1},\left(\begin{array}{cc}
\alpha_{1} \alpha_{1}^{\prime}+\Psi_{1} & \alpha_{1} \alpha_{2}^{\prime} \\
\alpha_{2} \alpha_{1}^{\prime} & \alpha_{2} \alpha_{2}^{\prime}+\Psi_{2}
\end{array}\right)\right)
$$

The conditional distribution $r_{1 t} \mid r_{2 t}, g_{1}$ is again normal with conditional mean

$$
\begin{aligned}
E\left(r_{1 t} \mid r_{2 t}, g_{t}\right) & =\phi_{1} g_{t}+\alpha_{1} \alpha_{2}{ }^{\prime}\left(\alpha_{2} \alpha_{2}{ }^{\prime}+\Psi_{2}\right)^{-1}\left(r_{2 t}-\phi_{2 g_{\imath}}\right) \\
& =\phi_{1}{ }^{*} g_{t}+\alpha_{1} \beta_{1}{ }^{\prime} r_{2 t}
\end{aligned}
$$

and the same conditional variance $\Lambda_{1.2}$. Under (9) one obtains $\phi_{1}=\alpha_{1} \delta$, $\phi_{2}=\alpha_{2} \delta$ which, substituting in the previous expression, implies

$$
\begin{aligned}
E\left(r_{1 t} \mid r_{2 t}, g_{t}\right) & =\alpha_{1}\left(I_{k}-\beta_{1}{ }^{\prime} \alpha_{2}\right) \delta g_{t}+\alpha_{1} \beta_{1}{ }^{\prime} r_{2 t}= \\
& =\alpha_{1}\left(\beta_{1}, \beta_{2}\right)\left(\begin{array}{l}
r_{2 t} \\
g_{t}
\end{array}\right)=\alpha_{1} \beta^{\prime}\left(\begin{array}{l}
r_{2 t} \\
g_{t}
\end{array}\right)
\end{aligned}
$$

where $\beta_{2}{ }^{\prime} \equiv\left(I_{k}-\beta_{1} \alpha_{2}\right) \delta$ is a $k \times s$ matrix.

Proof of corollary 5

Q.E.D.

We will sketch the proof for the analogue of (13); the analogue of (12) follows then by setting $g_{t}=1$. Let $\underline{m}_{t-1} \equiv\left(m_{t-1}, \ldots, m_{t-q}\right)^{\prime}, A^{*} \equiv\left(A_{1}, \ldots, A_{q}\right)$, $\underline{m}_{t}=\left(m_{t}{ }^{\prime}, \underline{m}_{t-1}\right)^{\prime}, A \equiv\left(I,-A^{*}\right)$. Note that $\underline{m}_{t-1}$ and $g_{t}$ may have elements in common, or even coincide. Let also $\Lambda \equiv\left(\alpha^{\prime}, \gamma^{\prime}\right)^{\prime}$. The distribution of $\left(r^{\prime}, m_{t}{ }^{\prime}\right)^{\prime}$ conditional on the past is

$$
\left(\begin{array}{c}
r_{t} \\
m_{t}
\end{array}\right) \mid m_{t-1}, g_{t} \sim N\left(\left(\begin{array}{c}
\phi g_{t} \\
A^{*} \underline{m}_{-1}
\end{array}\right), \Lambda \Lambda^{\prime}+\left(\begin{array}{cc}
\Psi & 0 \\
0 & \Sigma
\end{array}\right)\right)
$$

from which follows that the distribution of $r_{t} \mid m_{s}, g_{i}$ is normal with conditional expectation

$$
\begin{aligned}
E\left(r_{t} \mid \underline{m}_{,}, g_{t}\right) & =\alpha \gamma^{\prime}\left(\Sigma+\gamma^{\prime}\right)^{-1}\left(m_{t}-A^{*} \underline{m}_{t-1}\right)+\phi g_{t}= \\
& =\alpha \gamma^{\prime}\left(\Sigma+\gamma^{\prime}\right)^{-1}\left(m_{t}-A^{*} \underline{m}_{t-1}\right)+\alpha \delta g_{t}= \\
& =\alpha \beta_{l}^{\prime} \underline{m}_{t}+\alpha \beta_{2}^{\prime} g_{t}=\alpha \beta^{\prime}\left(m_{t}^{\prime}, g_{t}^{\prime}\right)^{\prime}
\end{aligned}
$$

Q.E.D.

Theorem A. 1

The minimum (maximum) of the function $f(x)=\left|x^{\prime} M x\right| /\left|x^{\prime} N x\right|$ is found by solving the eigenvalue problem $|\lambda N-M|=0$

\section{Proof [see e.g. Johansen (1989)]}

Since

$\ln (f(x))=\ln \left|x^{\prime} M x\right|-\ln \left|x^{\prime} N x\right|$

consider $\ln \left|x^{\prime} M x\right|$ and the increment $h$ of $x$; one has

$\ln \left|(x+h)^{\prime} M(x+h)\right|=\ln \left|\left(x^{\prime} M x\right) \quad\left(x^{\prime} M x\right)^{-1} \quad\left(x^{\prime} M x+x^{\prime} M h+h^{\prime} M x+h^{\prime} M h\right)\right|=$

$$
=\ln \left|x^{\prime} M x\right|+\ln \left|I+\left(x^{\prime} M x\right)^{-1}\left(x^{\prime} M h+h^{\prime} M x+h^{\prime} M h\right)\right|
$$

The last matrix on the right hand side is of the form $I-B\left(C+C^{\prime}+D\right)$. The matrix $A \equiv B^{1 / 2}\left(C+C^{\prime}+D\right) B^{1 / 2}$ is symmetric and can be decomposed as $V \Lambda V^{\prime}$. Since $\left|I+B\left(C+C^{\prime}+D\right) \models I I+A \models\right| I-V \Lambda V^{\prime} \models|I-\Lambda|$ one has

$\ln \left|(x+h)^{\prime} M(x+h)\right|=\ln \left|x^{\prime} M x\right|+\sum_{i=1}^{p} \ln \left(2+\lambda_{i}\right) \tilde{=} \ln \left|x^{\prime} M x\right|+\sum_{i=1}^{p} \lambda_{i}$ 
Now

$\operatorname{tr}(A)=\operatorname{tr}\left(B^{1 / 2}\left(C+C^{\prime}+D\right) B^{12,}\right)=\operatorname{tr}\left(B^{12} B^{12 \prime}\left(C+C^{\prime}+D\right)\right)=\operatorname{tr}\left(B\left(C+C^{\prime}+D\right)\right)=\operatorname{tr}(B C)+\operatorname{tr}\left(B C^{\prime}\right)+\operatorname{tr}(B D)$

Since $B$ is symmetric, $\operatorname{tr}\left(B C^{\prime}\right)=\operatorname{tr}\left(B^{\prime} C^{\prime}\right)=\operatorname{tr}\left(C^{\prime} B^{\prime}\right)=\operatorname{tr}(B C)$ and thus

$\ln \left|(x+h)^{\prime} M(x+h)\right|=\ln \left|x^{\prime} M x\right|+2 \operatorname{tr}(B C)+\operatorname{tr}(B D)$

For small values of $h$

$\ln \left|(x+h)^{\prime} M(x+h)\right|-\ln \left|x^{\prime} M x\right|=2 \operatorname{tr}\left(\left(x^{\prime} M x\right)^{-1}\left(x^{\prime} M h\right)+O\left(|h|^{2}\right)\right.$

Therefore the derivative with respect to $x$ is

$\frac{\partial \operatorname{tr}\left(\left(x^{\prime} M x\right)^{-1}\left(x^{\prime} M h\right)\right)}{\partial x^{\prime}}=2 M^{\prime} x\left(x^{\prime} M x\right)^{-1}$

and the derivative of $\ln f(x)$ is

$2 M^{\prime} x\left(x^{\prime} M x\right)^{-1}-2 N^{\prime} x\left(x^{\prime} N x\right)^{-1}$

Setting it to zero, the condition for $x$ to be a stationary point is

(A.1) $M^{\prime} x\left(x^{\prime} M x\right)^{-1}=N^{\prime} x\left(x^{\prime} N x\right)^{-1}$

The matrix of eigevalues $\Lambda$ and of eigenvectors $V$ of the problem $|\lambda N-M|=0$ satisfy

(A.2) $V \cdot M V=\Lambda$

(A.3) $V^{\prime} N V=I$

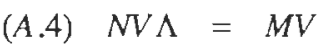

Substituting (A.2) into (A.4) one gets $N V\left(V^{\prime} M V\right)=M V$ or, from (A.3) $N V\left(V^{\prime} N V\right)^{-1}=M V\left(V^{\prime} M V\right)^{-1}$ thus showing that the matrix $V$ satisfies the first order condition (A.1). Therefore $x$ can be set equal to a suitable submatrix of $V$.
Table 1 - Number of significant returns autocorrelations for the 29 stocks; lags 1 to $12 ;+$ indicates positive autocorrelations greater than

$2 / \sqrt{n}$ and - indicates negative autocorrelations less than $2 / \sqrt{n}$.

\begin{tabular}{|c|c|c|c|c|c|c|c|c|c|c|c|c|}
\hline $\begin{array}{c}\operatorname{la} \\
\mathrm{g}\end{array}$ & 1 & 2 & 3 & 4 & 5 & 6 & 7 & 8 & 9 & 10 & 11 & 12 \\
\hline+ & 1 & 1 & 7 &. &. & 4 & 5 &. & 1 &. &. & 9 \\
\hline- & 1 &. &. &. &. &. &. & 2 &. & 1 &. &. \\
\hline
\end{tabular}

Table 2 - Wilk's $\lambda$ for the hypotheses $\Gamma=0, \Phi=0$ and $\Gamma=0=\Phi$ in $r_{t}=\mu+\Gamma r_{t-12}+\Phi D_{t}+\varepsilon_{t}, D_{t}$ are seasonal dummies

\begin{tabular}{|c|c|c|c|c|}
\hline \multicolumn{5}{|c|}{ sample period 1973.2 1989.12} \\
\hline Hypothesis & Wilks $\lambda$ & $F_{c}$ & $n, m$ & $\operatorname{Pr}\left(F>F_{c}\right)$ \\
\hline$\Gamma=0$ & 0.00448327 & 1.0377 & 841, & 0.2482 \\
$\Phi=0$ & 0.09443436 & 1.1399 & 2897.159 & 0.0627 \\
$\Gamma=0=\Phi$ & 0.00061840 & 1.0655 & 319, & 0.0917 \\
& & & 1414.019 & \\
& & & 1160, & \\
\hline \multicolumn{5}{|c|}{ sample period 1980.1 } \\
& & & 3345.959 & \\
\hline Hypothesis & Wilks $\lambda$ & $F_{c}$ & $n, m$ & $\operatorname{Pr}\left(F>F_{c}\right)$ \\
\hline$\Gamma=0$ & 0.00002209 & 0.9720 & 841, & 0.6706 \\
$\Phi=0$ & 0.00755709 & 1.0604 & 1192.622 & 0.2740 \\
$\Gamma=0=\Phi$ & 0.00000083 & 0.9791 & 319, & 0.6456 \\
& & & 558.1564 & \\
& & & $1160,1395.23$ & \\
\hline
\end{tabular}


Table 3 - Wilk's $\lambda$ for the hypotheses $\Gamma=0$ in $r_{t}=\mu+\Gamma D_{t}+\varepsilon_{t}$; sample period is 1972.21989 .12 .

\begin{tabular}{|l|c|c|c|c|}
\hline $\begin{array}{c}\text { monthly } \\
\text { dummies }\end{array}$ & Wilks $\lambda$ & $F_{c}$ & $n, m$ & $\operatorname{Pr}\left(F>F_{c}\right)$ \\
\hline January & 0.834994 & 1.1925 & 29,175 & 0.2421 \\
February & 0.893347 & 0.7294 & 29,175 & 0.8509 \\
March & 0.824142 & 1.2876 & 29,175 & 0.1628 \\
April & 0.804973 & 1.4620 & 29,175 & 0.0717 \\
June & 0.799139 & 1.5167 & 29,175 & 0.0542 \\
July & 0.821990 & 1.3068 & 29,175 & 0.1495 \\
August & 0.859814 & 0.9839 & 29,175 & 0.4957 \\
September & 0.879341 & 0.8280 & 29,175 & 0.7192 \\
October & 0.884143 & 0.7907 & 29,175 & 0.7686 \\
November & 0.868323 & 0.9151 & 29,175 & 0.5951 \\
December & 0.852155 & 1.0469 & 29,175 & 0.4091 \\
\hline \multicolumn{1}{|c|}{ all } & 0.162253 & 1.1106 & 319, & 0.1043 \\
& & & 1836.795 & \\
\hline
\end{tabular}

Table 4 - Likelihood ratio test in factor analysis.

\begin{tabular}{|c|c|c|c|c|}
\hline$\left.H_{0}: \quad k=j^{\mathrm{a}}\right)$ & $H_{A}: \quad k=p$ & $-2 \ln \lambda(j)$ & $d f$ & $\operatorname{Pr}\left(\chi^{2}>-2 \ln \lambda\right)$ \\
\hline 5 & 29 & 354.04 & 271 & 0.0005 \\
6 & 29 & 287.64 & 247 & 0.0386 \\
7 & 29 & 235.56 & 224 & 0.2849 \\
\hline$H_{0}: \quad k=j$ & $H_{A}: k=j+1$ & $-2 \ln \lambda(j)$ & $d f$ & $\operatorname{Pr}\left(\chi^{2}>-2 \ln \lambda\right)$ \\
\hline 5 & 6 & 66.40 & 24 & 0.0000 \\
6 & 7 & 52.08 & 23 & 0.0005 \\
7 & 29 & 235.56 & 224 & 0.2849 \\
\hline
\end{tabular}

a) Results have been obtained by SAS Factor procedure for the period 1980.02 . 1989.12.
Table 5 - Likelihood ratio test in reduced rank regression $r_{1 t}=\alpha \beta^{\prime}\left(r_{2}^{\prime}, 1\right)^{\prime}+\varepsilon_{t} ; n_{1}=15, n_{2}=14$.

\begin{tabular}{|c|c|c|c|c|c|}
\hline$H_{0}:$ & $k=j$ & $H_{A}: \quad k=p$ & $Q_{1}(j)$ & $v_{1}$ & $\operatorname{Pr}\left(\chi^{2}>Q_{1}\right)$ \\
\hline & $\begin{array}{c}0 \\
1 \\
2 \\
3 \\
4 \\
5 \\
5 \\
6 \\
7 \\
8 \\
9 \\
10 \\
11 \\
12 \\
13 \\
\end{array}$ & 15 & $\begin{array}{c}264.85047 \\
189.35026 \\
144.95116 \\
105.81639 \\
72.15284 \\
50.47726 \\
34.03070 \\
23.26108 \\
14.83220 \\
8.51379 \\
4.18512 \\
1.87613 \\
0.64080 \\
0.00302\end{array}$ & $\begin{array}{l}225 \\
196 \\
169 \\
144 \\
121 \\
100 \\
81 \\
64 \\
49 \\
36 \\
25 \\
16 \\
9 \\
4\end{array}$ & $\begin{array}{l}0.03524 \\
0.62018 \\
0.90974 \\
0.99279 \\
0.99987 \\
0.99999 \\
0.99999 \\
0.99999 \\
0.99999 \\
0.99999 \\
0.99999 \\
0.99999 \\
0.99991 \\
0.99999\end{array}$ \\
\hline$H_{0}:$ & $k=j^{\text {a) }}$ & $H_{A}: \quad k=j+1$ & $Q_{2}(j)$ & $v_{2}$ & $\operatorname{Pr}\left(\chi^{2}>Q_{2}\right)$ \\
\hline & $\begin{array}{l}0 \\
1 \\
2 \\
3 \\
4 \\
5 \\
6 \\
7 \\
8 \\
9 \\
10 \\
11 \\
12 \\
13 \\
\end{array}$ & $\begin{array}{c}1 \\
2 \\
3 \\
4 \\
5 \\
5 \\
6 \\
7 \\
8 \\
9 \\
10 \\
11 \\
12 \\
13 \\
14 \\
\end{array}$ & $\begin{array}{c}75.50021 \\
44.39910 \\
39.13477 \\
33.66355 \\
21.67558 \\
16.44655 \\
10.76962 \\
8.42888 \\
6.31842 \\
4.32866 \\
2.30899 \\
1.23533 \\
0.63777 \\
0.00302 \\
\end{array}$ & $\begin{array}{l}29 \\
27 \\
25 \\
23 \\
21 \\
19 \\
17 \\
15 \\
13 \\
11 \\
9 \\
7 \\
5 \\
3 \\
\end{array}$ & $\begin{array}{l}0.00001 \\
0.01880 \\
0.03571 \\
0.07018 \\
0.41841 \\
0.62731 \\
0.86829 \\
0.90544 \\
0.93379 \\
0.95930 \\
0.98559 \\
0.99009 \\
0.98621 \\
0.99996\end{array}$ \\
\hline
\end{tabular}


Table 6 - ADF unit root tests for some macroeconomic indicators; critical values $5 \%-2.891 \%-3.51$

\begin{tabular}{|l|c|c|c|}
\hline Variable & $\begin{array}{c}\text { Sample } \\
\text { period }\end{array}$ & \# lags & $t_{\alpha}$ \\
\hline INFL & $80.2 / 89.12$ & 4 & -1.86 \\
BOT & $81.2 / 89.12$ & 12 & -1.36 \\
BTP & $81.2 / 89.12$ & 12 & -1.41 \\
M2 & $81.2 / 89.12$ & 12 & 1.22 \\
PI & $73.2 / 88.12$ & 12 & -0.98 \\
BTP-BOT & $80.6 / 89.12$ & 4 & -3.03 \\
\hline
\end{tabular}

Table 7 - Likelihood ratio test in the reduced rank regression $r_{t}=\alpha \beta^{\prime}\left(h_{t}^{\prime}, 1\right)^{\prime}+\varepsilon_{t}$; the macroeconomic indicators are

$D B O T_{t}, D I N F L_{t}, D P I_{t}, D M 2_{\imath}, D D M K_{t}, D D O L L_{t}, D O I L_{i}, D M I B_{t}, D T U S_{t}, B O T-B T P_{t}$ $h_{i}$ is the residuals from a VAR; period is 1980.2 - 1989.12 .

\begin{tabular}{|c|c|c|c|}
\hline$H_{0}: k=j$ & $H_{A}: k=p$ & $Q_{1}(j)$ & $\operatorname{Pr}\left(\chi^{2}>Q_{1}\right)$ \\
\hline 0 & 10 & 460.7194 & 0.0000 \\
1 & & 360.6948 & 0.0001 \\
2 & & 279.8360 & 0.0022 \\
3 & & 216.8625 & 0.0395 \\
4 & & 159.6935 & 0.2788 \\
5 & & 110.2734 & 0.7265 \\
6 & & 78.9854 & 0.8312 \\
7 & & 49.4758 & 0.9357 \\
8 & & 25.8669 & 0.9761 \\
9 & & 11.8100 & 0.9224 \\
\hline & & & \\
$H_{0}: k=j$ & $H_{A}: k=j+1$ & $Q_{2}(j)$ & $\operatorname{Pr}\left(\chi^{2}>Q_{2}\right)$ \\
\hline 0 & 1 & 100.1246 & 0.0000 \\
1 & 2 & 80.8588 & 0.0000 \\
2 & 3 & 62.9735 & 0.0018 \\
3 & 4 & 57.1689 & 0.0040 \\
4 & 5 & 49.4201 & 0.0142 \\
5 & 6 & 31.2879 & 0.3044 \\
6 & 7 & 29.5096 & 0.2884 \\
7 & 8 & 23.6088 & 0.4841 \\
8 & 9 & 14.0569 & 0.8994 \\
9 & 10 & 11.8100 & 0.9224 \\
\hline
\end{tabular}

The value 0.0000 in this table and in the following ones indicates a probability value less than 0.0001 .
Table 8 - Specification tests for each macroeconomic indicator.

\begin{tabular}{|c|c|c|}
\hline Variable & ${ }_{H} Q_{3}^{\prime}(k=3)$ & $\operatorname{Prob}\left(\chi^{2}(3)>{ }_{H} Q_{3}\right)$ \\
\hline$D B O T_{i}$ & 16.5016 & 0.0024 \\
\hline$D I N F L_{t}$ & 6.4367 & 0.1688 \\
\hline$D P I_{s}$ & 7.9401 & 0.0938 \\
\hline$D M 2_{t}$ & 15.1580 & 0.0044 \\
\hline$D D M_{t}$ & 6.5976 & 0.1587 \\
\hline DUSD, & 5.6101 & 0.2302 \\
\hline DOIL & 6.7168 & 0.1516 \\
\hline$D M I B$ & 46.3810 & 0.0000 \\
\hline DTUS, & 9.2387 & 0.0554 \\
\hline$B O T-B T P_{t}$ & 11.0334 & 0.0262 \\
\hline
\end{tabular}

Table 9 - Likelihood ratio tests in the reduced rank regression $r_{t}=\alpha \beta^{\prime}\left(m^{\prime}, 1\right)^{\prime}+\varepsilon_{i}$; the variables in $m_{t}$ are the ones of table 7 .

\begin{tabular}{|c|c|c|c|c|}
\hline$H_{0}: k=j$ & $H_{A^{*}} k=n$ & $Q_{1}(j)$ & $v_{1}$ & $\operatorname{Pr}\left(\chi^{2}>Q_{1}\right)$ \\
\hline 0 & 29 & 1605.98842 & 1189 & 0.00000 \\
1 & & 1365.93758 & 1120 & 0.00000 \\
2 & & 1210.21703 & 1053 & 0.00045 \\
3 & & 1083.35388 & 988 & 0.01810 \\
4 & & 969.36870 & 925 & 0.15128 \\
5 & & 857.91884 & 864 & 0.55195 \\
6 & & 770.93611 & 805 & 0.80082 \\
7 & & 686.86042 & 748 & 0.94615 \\
8 & & 606.89865 & 693 & 0.99176 \\
9 & & 531.59932 & 640 & 0.99932 \\
\hline$H_{*}: k=j$ & $H_{i} k=j+1$ & $Q_{2}(j)$ & & \\
\hline 0 & 1 & 240.05084 & 69 & 0.00000 \\
1 & 2 & 155.72055 & 67 & 0.00000 \\
2 & 3 & 126.86315 & 65 & 0.00001 \\
3 & 4 & 113.98518 & 63 & 0.00009 \\
4 & 5 & 111.44986 & 61 & 0.00009 \\
5 & 6 & 86.98273 & 59 & 0.01035 \\
6 & 7 & 84.07569 & 57 & 0.01134 \\
7 & 8 & 79.96178 & 55 & 0.01561 \\
8 & 9 & 75.29933 & 53 & 0.02369 \\
9 & 10 & 63.27562 & 51 & 0.11613 \\
\hline
\end{tabular}




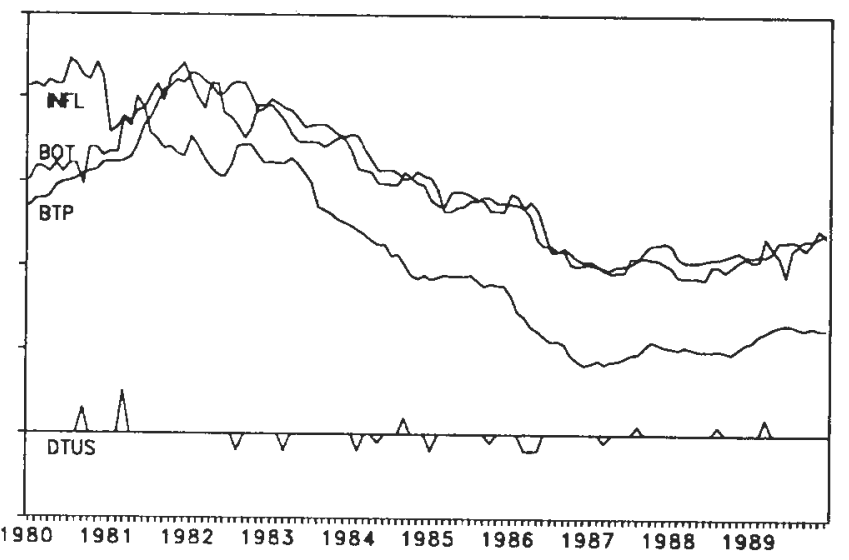

Fig. 1 - Interest and inflation rate, January 1980 - December 1989.

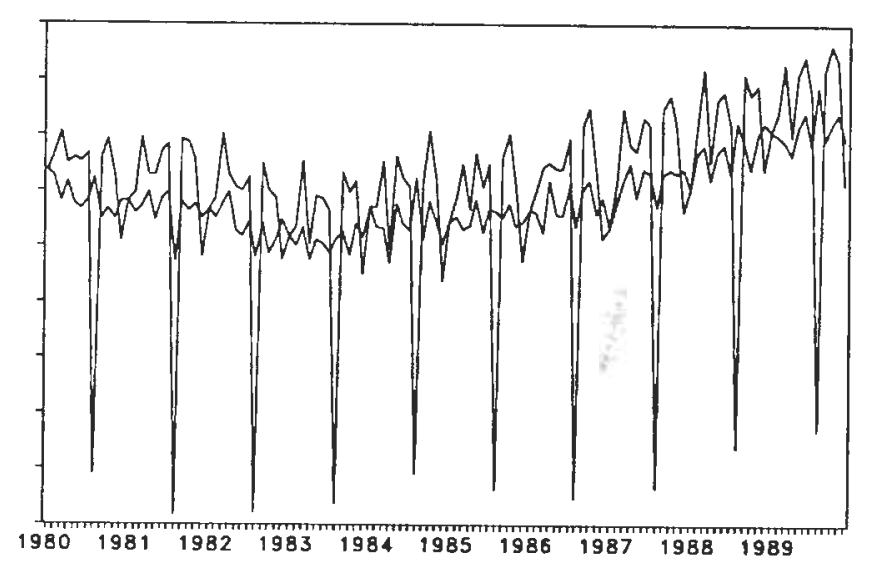

Fig. 2 - Industrial production index, seasonally adjusted and unadjusted January 1980 - December 1989.

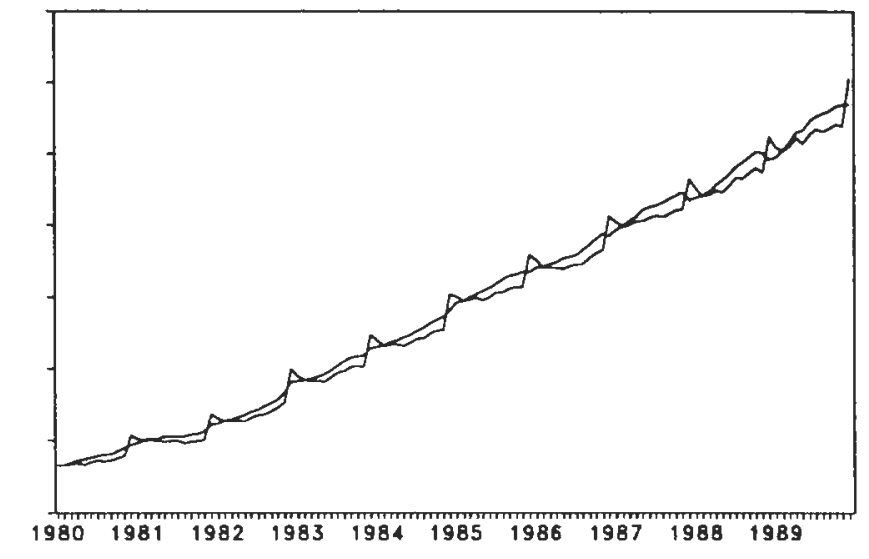

Fig. 3 - $\ln (M 2) ;$ seasonally adjusted and unadjusted January 1980 . December 1989. 